\section{Diálise planejada e a utilização regular da atenção primária à saúde entre os pacientes diabéticos do Município de Belo Horizonte, Minas Gerais, Brasil}

\author{
Planned dialysis and regular use of primary care \\ by diabetic patients in the city of Belo Horizonte, \\ Minas Gerais State, Brazil
}

Diálisis planificada y el uso regular de la atención primaria de la salud entre los pacientes diabéticos en la ciudad de Belo Horizonte, Minas Gerais, Brasil
Eline Rezende de Morais Peixoto ${ }^{1}$ Ilka Afonso Reis 1

Elaine Leandro Machado 2

Eli Iola Gurgel Andrade 1

Francisco de Assis Acurcio 1 Mariângela Leal Cherchiglia ${ }^{1}$

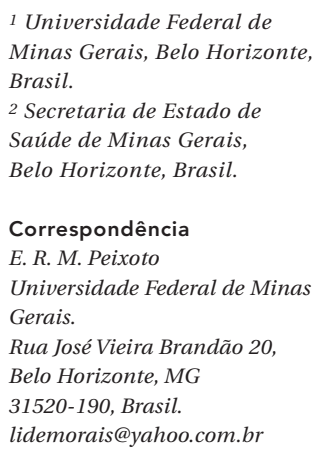

1 Universidade Federal de Minas Gerais, Belo Horizonte, Brasil.

2 Secretaria de Estado de Saúde de Minas Gerais, Belo Horizonte, Brasil.

Correspondência E. R. M. Peixoto Universidade Federal de Minas Gerais.

Rua José Vieira Brandão 20, Belo Horizonte, $M G$ 31520-190, Brasil. lidemorais@yahoo.com.br

\section{Abstract}

The objective was to analyze factors associated with planned initiation of dialysis in diabetic patients in the city of Belo Horizonte, Minas Gerais State, Brazil. This was a cross-sectional study of 250 diabetic patients who began dialysis between January 2006 and December 2007. Initiating dialysis with an arteriovenous fistula or in peritoneal dialysis was classified as planned initiation. The study targeted socio-demographic, clinical, and health services use variables using a semi-structured interview. Multivariate analysis used Poisson regression. Seventy per cent of the patients began dialysis on an unplanned basis, and 67\% of those consulted had been referred to a nephrologist more than four months previously. Attending a health center, not having the first nephrology appointment paid for by the Brazilian Unified National Health System, and having a choice of treatment for the kidney disease were associated with planned initiation of dialysis. Unplanned initiation of dialysis is common in the city of Belo Horizonte and occurs regardless of the time since referral to the nephrologist.

Chronic Kidney Failure; Diabetes Mellitus; Dialysis; Health Evaluation; Cross-Sectional Studies

\section{Resumo}

O objetivo foi de analisar os fatores associados ao início planejado da diálise dos pacientes diabéticos que iniciaram o tratamento no Município de Belo Horizonte, Minas Gerais, Brasil. Estudo transversal com 250 pacientes diabéticos que iniciaram diálise entre janeiro de 2006 e dezembro de 2007. Iniciar a diálise com fístula arteriovenosa ou em diálise peritoneal foi classificado como início planejado. Foram investigadas as variáveis sociodemográficas, clínicas e de utilização de serviços de saúde por meio de entrevista semiestruturada. Para análise multivariada utilizou-se regressão de Poisson. Setenta por cento dos pacientes começaram a diálise de forma não planejada e 67\% dos que consultaram com o nefrologista foram encaminhados com mais de quatro meses. Frequentar o centro de saúde, não ter a primeira consulta com nefrologista paga pelo SUS e ter tido opção de escolha para o tratamento da doença renal foram fatores relacionados ao início planejado da diálise. O início não planejado da diálise é comum no Município de Belo Horizonte e ocorre independentemente do tempo de encaminhamento ao nefrologista.

Falência Renal Crônica; Diabetes Mellitus; Diálise; Avaliação em Saúde; Estudos Transversais 


\section{Introdução}

A doença renal crônica é a perda progressiva e irreversível da função renal e, em sua fase mais avançada, a doença renal crônica terminal, o paciente necessita de uma terapia renal substitutiva (TRS) 1. Atualmente, a doença renal crônica terminal é um dos maiores desafios à saúde pública devido às suas implicações econômicas e sociais 2,3 , potencializadas quando se considera que, na maioria dos casos, a perda da função renal poderia ser evitada com a correta abordagem clínica da doença renal crônica 4 .

A relevância do problema para o Sistema Único de Saúde (SUS) é reconhecida por meio da publicação da Portaria no 1168/GM em 5 de junho de $20044^{5}$, que institui a Política Nacional da Atenção ao Portador de Doença Renal, e define estratégias assistenciais estruturadas na busca por um cuidado equânime e qualificado dos pacientes com doença renal crônica incluindo prevenção, promoção, tratamento e reabilitação, como resultado da articulação entre os vários níveis de atenção à saúde do SUS.

Em 2011, segundo o censo da Sociedade Brasileira de Nefrologia, havia um total de 91.314 pacientes em tratamento dialítico no Brasil, sendo o diabetes mellitus a segunda causa de doença renal crônica terminal para $28,4 \%$ dos pacientes em diálise ${ }^{6}$. Em face da crescente incidência da doença renal crônica terminal causada pelo diabetes mellitus ${ }^{6}$, o enfoque preventivo e terapêutico no tratamento do diabetes é necessário com o objetivo de garantir melhores resultados na prevenção da doença renal crônica.

No Brasil, a atenção primária à saúde tem caráter estratégico na realização de ações de promoção, vigilância em saúde, prevenção, assistência e acompanhamento longitudinal dos portadores de doenças crônicas não transmissíveis, sendo responsável pelo encaminhamento precoce dos pacientes com doença renal crônica para a atenção especializada de média complexidade, onde será instituída a terapêutica apropriada para retardar a progressão da doença renal crônica, prevenir suas complicações e garantir um preparo adequado para a iniciação às terapias de substituição renal 1,5,7,8.

No entanto, diversos estudos verificaram falhas no manejo clínico de pacientes com doença renal crônica, resultando no encaminhamento tardio ao nefrologista e início não planejado da diálise 9,10,11. O encaminhamento tardio ao nefrologista mostrou-se associado ao maior uso de cateter para o início da hemodiálise, maior mortalidade e menor possibilidade de escolha da modalidade da diálise 12,13,14. Por outro lado, o encaminhamento precoce resulta em uma me- lhor condição clínica e melhor preparação para o início da diálise, pois aumenta a probabilidade dos pacientes iniciarem a diálise de forma planejada, em serviços ambulatoriais, com a fístula arteriovenosa madura ou na modalidade de diálise peritoneal 12,15,16,17. Contudo, existem poucos estudos brasileiros sobre a qualidade do tratamento ofertado aos pacientes com doença renal na fase pré-dialítica e o processo de cuidado no nível primário de assistência à saúde 18,19.

A análise do cuidado pré-diálise dos pacientes diabéticos se justifica tendo em vista que o diabetes mellitus é um fator de risco potencial para a doença renal crônica terminal, além de ser responsável por uma parcela importante dos pacientes em terapia renal substitutiva. Ademais, as ações integradas de prevenção e cuidado ao diabetes mellitus ocorrem prioritariamente na atenção primária, por meio de protocolos de cuidado específicos, o que torna possível analisar aspectos importantes do cuidado ofertado no período que antecedeu a diálise, relacionando-o com o modo como se iniciou a terapia dialítica daqueles que começaram o tratamento dialítico no período de janeiro de 2006 a dezembro de 2007, no Município de Belo Horizonte, Minas Gerais, Brasil.

\section{Materiais e métodos}

Estudo transversal que utiliza dados coletados nas 12 unidades prestadoras de diálise que atendem pelo SUS, existentes na cidade de Belo Horizonte. Este trabalho faz parte de um estudo mais amplo, denominado Avaliação Econômica e Epidemiológica das Terapias de Substituição Renal no Brasil - Projeto TRS, realizado pelo Grupo de Pesquisa em Economia da Saúde, Universidade Federal de Minas Gerais (GPES-UFMG).

No projeto original foram incluídos todos os pacientes maiores de 18 anos, que iniciaram tratamento dialítico entre janeiro de 2006 e dezembro de 2007 em serviços de diálise credenciados pelo SUS em Belo Horizonte, com no mínimo três meses de tratamento, sem histórico de realização de transplante renal anterior a janeiro de 2007 e que concordaram em participar da pesquisa. Foram excluídos os pacientes que não tinham condições de responder ao questionário (dificuldade de compreensão das questões, deficiência visual ou auditiva); que se recusaram a responder à triagem dos critérios de inclusão; pacientes ausentes, após três tentativas de abordagem; pacientes internados ou que receberam alta da diálise (considerados como insuficiência renal aguda); que foram transferidos para acompanhamento da doença renal crônica terminal 
em outra cidade ou sem identificação do local de transferência; e pacientes que responderam menos de $50 \%$ do questionário proposto.

Os dados primários foram obtidos pela realização de entrevistas em duas etapas. Na la Fase, foram coletados os dados retrospectivos relativos aos 2 anos de tratamento em TRS anteriores a 2008, e na 2a os dados relativos ao desfecho dos pacientes participantes da 1a Fase, com seguimento prospectivo de 1,5 ano. Dados de prontuários dos pacientes que aceitaram participar da pesquisa (1a Fase) e de pacientes que foram a óbito antes do início do estudo, mas que atendiam aos critérios de inclusão, segundo informações dos serviços de diálise participantes, também foram coletados para levantamento de variáveis clínicas de interesse.

Os dados sociodemográficos, de gastos, econômicos e clínicos foram obtidos por meio de questionário pré-testado e validado em estudo piloto. Para avaliação da qualidade de vida foram utilizados os instrumentos: KDQOL-SF (Kidney Disease and Quality of Life, Short Form), SF-36 (Medical Outcome Survey-Short Form 36) e EQ-5D (EuroQol). Esses instrumentos são traduzidos e validados para serem aplicados na população brasileira 20,21.

Para avaliar a confiabilidade da entrevista, uma amostra de $5 \%$ dos entrevistados foi selecionada aleatoriamente para reentrevista sete dias após a primeira. Todos os dados de autorrelato incluídos na presente análise apresentaram boa confiabilidade.

Dos 747 pacientes que cumpriam os critérios de elegibilidade $34(4,5 \%)$ se recusaram a responder ao questionário. Não existe diferença entre os participantes das entrevistas e os que se recusaram em relação às variáveis do estudo. Os dados utilizados neste trabalho são referentes aos obtidos na linha de base (1a Fase).

Nesta análise, para a composição da variável resposta diálise planejada, foi utilizada a definição de Górriz et al. 22, que considera como planejada a hemodiálise iniciada com fístula arteriovenosa ou diálise peritoneal e, como não planejada a hemodiálise iniciada por meio de cateter. As variáveis explicativas foram: (i) socioeconômicas e demográficas: idade; sexo; cor da pele; estado civil; classe econômica, segundo a classificação da Associação Brasileira de Empresas de Pesquisa (ABEP) 23, que utiliza o grau de instrução do chefe da família e a posse de itens para diferenciar a população em cinco classes (A, melhor estado, B, C, D e E, pior estado); escolaridade (em anos de estudos) e cobertura de plano privado/convênio de saúde; (ii) clínicas: tempo de diagnóstico da doença renal crônica e diagnóstico de hipertensão arterial; e (iii) utilização de serviços de saú- de: uso regular de serviço de saúde e centro de saúde do SUS antes do início da diálise; acesso à informação sobre os riscos do diabetes mellitus evoluir para doença renal crônica; solicitação de exames para avaliar a função renal antes do início da diálise; consulta com nefrologista; serviço de saúde que realizou encaminhamento para o nefrologista; primeira consulta com nefrologista paga pelo SUS; tempo entre a consulta com nefrologista e início da diálise; serviço de saúde que diagnosticou a necessidade de diálise e a opção de escolha da modalidade de diálise (hemodiálise ou diálise peritoneal).

Foi realizada análise descritiva das variáveis por meio da distribuição de frequências, medidas de tendência central e de dispersão. Na análise univariada, o teste qui-quadrado de Pearson foi utilizado para comparar proporções entre os grupos de pacientes de acordo com o início da diálise: planejado e não planejado, considerando o nível de $5 \%$ de significância. Todos os testes de hipóteses foram do tipo bicaudal.

Para avaliar o efeito independente das variáveis explicativas na prevalência de diálise planejada, foi utilizado o modelo de regressão multivariado de Poisson com estimador de variância robusto. Além de permitir a interpretação dos resultados em termos da prevalência do evento, o uso do modelo de regressão de Poisson é preferível ao modelo de regressão logística usual quando a prevalência do evento é de moderada a alta ${ }^{24}$. Foi testada a hipótese de associação entre a variável resposta e diversas covariáveis que poderiam atuar como fatores de confusão. No entanto, não se obteve nenhum resultado estatisticamente significante. Para a seleção do modelo final foi utilizada a estratégia Backward, na qual todas as variáveis explicativas são incluídas no modelo inicial, sendo realizado o procedimento de retirada manual daquelas consideradas não significantes, até permanecerem no modelo final somente as variáveis que apresentam coeficientes estatisticamente significantes no nível de 5\% de significância. A adequação do modelo foi avaliada pelo teste da deviance. A análise dos dados foi realizada no programa SPSS versão 19 (SPSS Inc., Chicago, Estados Unidos).

Este projeto foi aprovado pelo Comitê de Ética em Pesquisa da UFMG (parecer ETIC 492/2006). Todos os participantes do estudo assinaram o termo de consentimento livre e esclarecido.

\section{Resultados}

Do total de pacientes entrevistados $(n=713), 250$ $(35,06 \%)$ relataram ter diabetes antes do início da diálise. Entre aqueles que disseram ter diag- 
nóstico de diabetes antes do início da diálise, 238 pacientes $(95,2 \%)$ iniciaram em hemodiálise e $12(4,8 \%)$ em diálise peritoneal. Dentre os que iniciaram em hemodiálise, 63 (26,5\%) pacientes utilizaram a fístula arteriovenosa como acesso. A maioria (70\%) iniciou a diálise de forma não planejada. Relataram mudança na modalidade inicial de diálise 14 (5,6\%) pacientes, sendo que 9 destes tinham iniciado na modalidade hemodiálise e 05 em diálise peritoneal. Observa-se uma predominância do sexo masculino $(54,8 \%)$, com idade média de 58,97 anos ( $\mathrm{dp}=13,01$ anos), com até oito anos de estudos $(68,4 \%)$, que pertenciam às classes econômicas C, D e E $(66,4 \%)$ e que não possuíam plano de saúde (56\%). A maioria $(58,8 \%)$ tinha doença renal crônica há mais de 1 ano, 96,1\% também relataram diagnóstico de hipertensão anterior à terapia renal substitutiva, frequentavam regularmente algum serviço de saúde $(78,3 \%)$, sendo que 120 pacientes disseram frequentar algum centro de saúde. Nesses serviços, a maioria foi informada pelo médico que o diabetes poderia causar doença renal crônica $(73,1 \%)$, além de ter a função renal avaliada antes de iniciar a diálise $(80,9 \%)$ A maioria havia consultado com um médico nefrologista antes do início da diálise (83,6\%), sendo encaminhados pelo médico que acompanhava o tratamento da hipertensão ou diabetes (54,3\%) e, para $33,6 \%$ dos pacientes, a primeira consulta com este especialista foi paga pelo SUS. Grande parte dos pacientes que consultou com o nefrologista foi encaminhada com mais de 4 meses $(67 \%)$. No entanto, a maioria $(61,6 \%)$ foi informada, pela primeira vez, da necessidade de realizar diálise em um serviço de urgência/hospital e $71 \%$ não tiveram opção de escolha para a modalidade de tratamento dialítico antes de iniciar a diálise.

A Tabela 1 apresenta o resumo das características sociodemográficas, clínicas e de utilização dos serviços de saúde, segundo o planejamento da diálise. Foi observado um porcentual maior de diálise planejada para pacientes com menos de 8 anos de estudos, pertencentes às classes econômicas A e B, que possuíam plano de saúde, tempo de doença renal crônica maior que 1 ano, além de terem sido informados pelo médico que o diabetes poderia causar doença renal crônica e realizado consulta com nefrologista. Houve um maior porcentual de não planejamento para aqueles que não tiveram opção de escolher a modalidade inicial da diálise.

No modelo multivariado final, foi observado que utilizar regularmente o centro de saúde do SUS, não ter a primeira consulta com o nefrologista paga pelo SUS e ter tido a opção de escolha da modalidade de diálise foram fatores relacionados ao início planejado do tratamento dialítico (Tabela 2).

A prevalência de pacientes diabéticos que começaram a diálise de forma planejada foi $13,5 \%$ menor para os pacientes que não tiveram opção de escolha entre hemodiálise e diálise peritoneal $(\mathrm{RP}=0,865)$; $18,9 \%$ menor para os que não frequentavam regularmente um centro de saúde $(\mathrm{RP}=0,811)$; e 29,3\% maior para aqueles que não tiveram a primeira consulta com um médico nefrologista paga pelo SUS $(R P=1,293)$.

\section{Discussão}

O presente estudo evidencia que o início não planejado da diálise é comum no Município de Belo Horizonte e ocorre independentemente do tempo de encaminhamento ao nefrologista. Resultados semelhantes foram encontrados em países como Espanha e Canadá 9,11. O cuidado nefrológico insuficiente, a falta de recursos dos centros de diálise para acomodar novos pacientes e dificuldades de acesso aos recursos cirúrgicos para confecção da fístula arteriovenosa foram citados como possíveis causas 11. Ademais, no Canadá houve relação entre o baixo número de cirurgiões vasculares com uma maior proporção de pacientes iniciando a hemodiálise com cateter temporário 25.

Observou-se a predominância da hemodiálise como modalidade inicial, sendo o cateter a via de acesso mais frequente. Esses dados são semelhantes aos encontrados em outros trabalhos realizados no Brasil 26,27. Andrade et al. 27 observaram que a alocação dos pacientes nas diferentes modalidades de diálise não foi aleatória, sendo que pacientes diabéticos, jovens e idosos tiveram maior probabilidade de serem alocados na modalidade diálise peritoneal. $\mathrm{Ou}-$ tros fatores como as condições clínicas, autonomia do paciente, educação e treinamento de médicos e enfermeiros, bem como fatores não clínicos, tais como financiamento, reembolso, disponibilidade de recursos e hábitos socioculturais influenciaram na alocação do paciente 28. Também podem interferir na escolha da diálise peritoneal a orientação dada pelo médico da atenção primária ou nefrologista, a distância da unidade dialisadora e atributos individuais, tais como idade, escolaridade, profissão e a presença de comorbidades 29

Por outro lado, o tipo de acesso inicial utilizado para a diálise pode ser considerado como uma forma indireta de avaliação do cuidado prédiálise 25. Começar a terapia com fístula arteriovenosa ou em diálise peritoneal significa que houve um tempo maior de preparo do paciente 
Tabela 1

Características dos pacientes diabéticos de acordo com o início da diálise planejada ou não planejada. Projeto TRS, Belo Horizonte, Minas Gerais, Brasil, 2006-2007.

\begin{tabular}{|c|c|c|c|c|}
\hline Variáveis & $\begin{array}{l}\text { Total } \\
\text { n (\%) }\end{array}$ & $\begin{array}{c}\text { Diálise } \\
\text { planejada } \\
\begin{array}{c}(\mathrm{n}=75) \\
\mathrm{n}(\%)\end{array}\end{array}$ & $\begin{array}{l}\text { Diálise não } \\
\text { planejada } \\
\begin{array}{c}\text { (n= 175) } \\
\text { n (\%) }\end{array}\end{array}$ & Valor de $p$ \\
\hline Sociodemográficas e clínicas & & & & 0,279 \\
\hline \multicolumn{5}{|l|}{$\operatorname{Sexo}(n=250)$} \\
\hline Masculino & $137(54,8)$ & $45(60,0)$ & $92(52,6)$ & \\
\hline Feminino & $113(45,2)$ & $30(40,0)$ & $83(47,4)$ & \\
\hline Cor da pele $(n=250)$ & & & & 0,298 \\
\hline Branca & $88(35,2)$ & $30(40,0)$ & $58(33,1)$ & \\
\hline Não branca & $162(64,8)$ & $45(60,0)$ & $117(66,9)$ & \\
\hline Idade $(n=250)$ (anos) & & & & 0,689 \\
\hline Média (DP) & $58,97(13,02)$ & $60(13,0)$ & $59(13,0)$ & \\
\hline Mediana & 60,0 & 60,0 & 60,0 & \\
\hline $18-40$ & $25(10,0)$ & $8(10,7)$ & $17(9,7)$ & \\
\hline $41-59$ & $90(36,0)$ & $24(32,0)$ & $66(37,7)$ & \\
\hline$>60$ & $135(54,0)$ & $43(57,3)$ & $92(52,6)$ & \\
\hline Escolaridade $(n=247)$ (anos) & & & & 0,013 \\
\hline$<8$ & $169(68,4)$ & $43(57,3)$ & $126(73,3)$ & \\
\hline$>8$ & $78(31,6)$ & $32(42,7)$ & $46(26,7)$ & \\
\hline Classe econômica $(n=238)$ & & & & 0,020 \\
\hline$A \in B$ & $80(33,6)$ & $31(43,1)$ & $49(29,5)$ & \\
\hline C & $103(43,3)$ & $32(44,4)$ & $71(42,8)$ & \\
\hline$D$ e $E$ & $55(23,1)$ & $9(12,5)$ & $46(27,7)$ & \\
\hline Plano de saúde $(n=250)$ & & & & 0,026 \\
\hline Sim & $110(44,0)$ & $41(54,7)$ & $69(39,4)$ & \\
\hline Não & $140(56,0)$ & $34(45,3)$ & $106(60,6)$ & \\
\hline Tempo de doença renal crônica $(n=250)$ (anos) & & & & 0,013 \\
\hline$\leq 1$ & $103(41,2)$ & $22(29,3)$ & $81(46,3)$ & \\
\hline$>1$ & $147(58,8)$ & $53(70,7)$ & $94(53,7)$ & \\
\hline Relata diagnóstico de hipertensão arterial antes da TRS $(n=250)$ & & & & 0,845 \\
\hline $\operatorname{Sim}$ & $228(91,2)$ & $68(90,7)$ & $160(91,4)$ & \\
\hline Não & $22(8,8)$ & $7(9,3)$ & $15(8,6)$ & \\
\hline \multicolumn{5}{|l|}{ Utilização de serviço de saúde antes da TRS } \\
\hline Utilização regular de serviço de saúde $(n=249)$ & & & & 0,153 \\
\hline $\operatorname{Sim}$ & $195(78,3)$ & $63(84,0)$ & $132(75,9)$ & \\
\hline Não & $54(26,9)$ & $12(16,0)$ & $42(24,1)$ & \\
\hline Utilização regular do centro de saúde do SUS $(n=194)$ & & & & 0,117 \\
\hline $\operatorname{Sim}$ & $120(61,9)$ & $34(54,0)$ & $86(65,6)$ & \\
\hline Não & $74(38,1)$ & $29(46,0)$ & $45(34,4)$ & \\
\hline Informado que diabetes mellitus poderia causar doença renal crônica $(n=245)$ & & & & 0,010 \\
\hline Sim & $179(73,1)$ & $63(84,0)$ & $116(68,2)$ & \\
\hline Não & $66(26,9)$ & $12(16,0)$ & $54(31,8)$ & \\
\hline Solicitação de exames para avaliar função renal $(n=241)$ & & & & 0,127 \\
\hline Sim & $195(80,9)$ & $65(86,7)$ & $130(78,3)$ & \\
\hline Não & $46(19,1)$ & $10(13,3)$ & $36(21,7)$ & \\
\hline
\end{tabular}

(continua) 
Tabela 1 (continuação)

\begin{tabular}{|c|c|c|c|c|}
\hline Variáveis & $\begin{array}{l}\text { Total } \\
\text { n (\%) }\end{array}$ & $\begin{array}{c}\text { Diálise } \\
\text { planejada } \\
\text { (n = 75) } \\
\text { n (\%) }\end{array}$ & $\begin{array}{l}\text { Diálise não } \\
\text { planejada } \\
\begin{array}{c}\text { (n = 175) } \\
\text { n (\%) }\end{array}\end{array}$ & Valor de $\mathrm{p}$ \\
\hline \multicolumn{5}{|l|}{ Utilização de serviço de saúde antes da TRS } \\
\hline Consultou com nefrologista $(n=241)$ & & & & 0,053 \\
\hline Sim & $209(86,7)$ & $68(93,2)$ & $141(83,9)$ & \\
\hline Não & $32(13,3)$ & $5(6,8)$ & $27(16,1)$ & \\
\hline Qual o serviço de saúde encaminhou para o nefrologista $(n=237)$ & & & & 0,166 \\
\hline Urgência/Hospital & $51(27,7)$ & $14(21,5)$ & $37(31,1)$ & \\
\hline Atenção primária & $133(72,3)$ & $51(78,5)$ & $82(68,9)$ & \\
\hline Primeira consulta com nefrologista paga pelo SUS $(n=178)$ & & & & 0,072 \\
\hline Sim & $84(47,2)$ & $24(38,1)$ & $60(52,2)$ & \\
\hline Não & $94(52,8)$ & $39(61,9)$ & $55(47,8)$ & \\
\hline \multicolumn{5}{|l|}{ Tempo entre a consulta com nefrologista e início da diálise $(n=164)$ (meses) } \\
\hline$<1$ & $30(18,3)$ & $7(14,0)$ & $23(20,2)$ & \\
\hline $2-4$ & $24(14,6)$ & $5(10,0)$ & $19(16,7)$ & \\
\hline $5-12$ & $36(22,0)$ & $10(20,0)$ & $26(22,8)$ & \\
\hline$>12$ & $74(45,1)$ & $28(56,0)$ & $46(40,4)$ & \\
\hline Serviço de saúde onde soube que precisava fazer diálise $(n=250)$ & & & & 0,002 \\
\hline Urgência/Hospital & $120(68,6)$ & $34(45,3)$ & $154(61,6)$ & \\
\hline PSF/PAM & $29(16,6)$ & $18(24,0)$ & $47(18,8)$ & \\
\hline Clínica particular/Outros & $26(14,9)$ & $23(30,7)$ & $49(19,6)$ & \\
\hline \multicolumn{5}{|l|}{ peritoneal) $(n=248)$} \\
\hline Sim & $72(29,0)$ & $31(41,3)$ & $41(23,7)$ & \\
\hline Não & $176(71,0)$ & $44(58,7)$ & $132(76,3)$ & \\
\hline
\end{tabular}

DP: desvio-padrão; PAM: Posto de Atendimento Médico; PSF: Programa Saúde da Família; TRS: terapia renal substitutiva.

Projeto TRS: Avaliação Econômica e Epidemiológica das Terapias de Substituição Renal no Brasil - Projeto TRS.

Tabela 2

Modelo de regressão de Poisson para o início planejado da diálise segundo variáveis socioeconômicas, clínicas e de utilização do serviço de saúde para os pacientes diabéticos. Belo Horizonte, Minas Gerais, Brasil, 2006-2007.

\begin{tabular}{|c|c|c|c|}
\hline Variáveis & RP & IC95\% & Valor de $p$ \\
\hline \multicolumn{4}{|c|}{ Utilização regular do centro de saúde do SUS antes da TRS } \\
\hline Sim & 1,000 & - & \\
\hline Não & 0,811 & $0,687-0,957$ & 0,013 \\
\hline \multicolumn{4}{|c|}{ Primeira consulta com nefrologista paga pelo SUS } \\
\hline Sim & 1,000 & - & \\
\hline Não & 1,293 & $1,098-1,524$ & 0,002 \\
\hline \multicolumn{4}{|c|}{ Teve opção de escolha da modalidade de diálise (hemodiálise ou diálise peritoneal) } \\
\hline Sim & 1,000 & - & \\
\hline Não & 0,865 & $0,757-0,989$ & 0,034 \\
\hline
\end{tabular}

IC95\%: intervalo de 95\% de confiança; TRS: terapia renal substitutiva. 
para a entrada em diálise, além de um processo de educação para escolha da modalidade de terapia renal substitutiva. No Brasil, no ano de 2007, foi observado que aproximadamente $70 \%$ dos pacientes iniciaram a hemodiálise com cateter venoso, sendo a baixa probabilidade de ter acesso vascular arteriovenoso como primeiro tipo de acesso, associada ao cuidado pré-diálise 30 . Resultado semelhante foi observado no Canadá, onde $70 \%$ dos pacientes incidentes na terapia renal substitutiva utilizaram cateter 25 . Nos países europeus, o uso de fístula arteriovenosa entre os incidentes em hemodiálise alcançou 50\%, sendo superior ao dos Estados Unidos, onde o porcentual ficou em torno de 18\% 31. Dificuldades de interação entre especialidades clínicas, como médico da família, nefrologista e cirurgião vascular em relação ao tempo de encaminhamento e o início da diálise, além da disponibilidade de recursos para a saúde, podem explicar o padrão de uso de cateter nas diferentes populações 25 .

Houve maior prevalência de planejamento da diálise entre os pacientes que relataram frequentar regularmente o centro de saúde. Sendo o diabetes a segunda causa de doença renal crônica no Brasil 6,26, o cuidado desses pacientes dependerá cada vez mais dos cuidados na atenção primária 32. Atualmente, o Município de Belo Horizonte conta com 147 centros de saúde, 523 equipes de saúde da família com índice de cobertura de $75 \% 33,34$, o que evidencia a importância desse nível de atenção na realização do diagnóstico precoce e encaminhamento para a média complexidade.

No entanto, Machado et al. 35 apontaram problemas na rede de atenção primária de Belo Horizonte relacionados ao cuidado do doente renal, como a falta de capacitação dos profissionais da atenção básica para detecção precoce, acompanhamento, monitoramento e controle dos principais grupos de risco para a doença renal, além de escassas medidas de promoção da saúde e prevenção da insuficiência renal.

Considerando a utilização de serviços de saúde pelos pacientes diabéticos, observou-se que a maioria foi encaminhada para consulta com o nefrologista pelo médico que acompanhava o tratamento do diabetes mellitus. Além disso, grande parte consultou com o nefrologista e realizou exames para verificar a função renal antes da diálise. O tempo entre a consulta com o nefrologista e o início da diálise foi maior que 4 meses para $67 \%$ dos pacientes. Analisando o tempo de encaminhamento, esperava-se que um número maior de pacientes iniciasse a hemodiálise por meio da fístula arteriovenosa, tendo em vista que diversos estudos verificaram que o encaminhamento precoce estava associado à maior utiliza- ção deste tipo de acesso 12,36,37,38. No entanto, a grande proporção de pacientes com início não planejado da diálise e daqueles que ficaram sabendo em um serviço hospitalar sobre a necessidade de terapia renal substitutiva podem indicar falhas no cuidado integral ao paciente renal crônico, assim como denotam dificuldades de acesso aos serviços assistenciais de média complexidade e/ou a baixa qualidade das ações assistenciais ofertadas neste nível de atenção. Fatores individuais como a baixa aderência ao tratamento e a resistência para a mudança de hábitos de vida também devem ser considerados 11 .

Os resultados encontrados também indicam uma fragilidade do mecanismo de referência e contrarreferência que prejudica o cuidado integral do paciente renal crônico, tendo em vista que grande parte deles em Belo Horizonte não tem sua primeira consulta com o nefrologista paga pelo SUS. Considerando que esses pacientes pertencem predominantemente às classes econômicas mais baixas e a maioria não possui plano de saúde, a continuidade da assistência especializada por meio do pagamento pode ter sido prejudicada.

A dificuldade de acesso à atenção especializada no Município de Belo Horizonte já foi citada como problema da rede SUS 4,39,40. Essa falta de coordenação entre os níveis de atenção da rede pública tem como consequência a desarticulação das ações de promoção da saúde, cuidados preventivos, curativos e reabilitadores, rompendo com o conceito de integralidade 39 .

Também foi observada uma menor prevalência de planejamento da diálise para os pacientes que não tiveram opção de escolher a modalidade inicial da diálise. Segundo Górriz et al. 22, Finkelstein et al. ${ }^{41}$ e Marrón et al. 9, o início não planejado da diálise implica a falta de informação e educação para o paciente sobre as opções disponíveis para a terapia renal substitutiva. $\mathrm{Na}$ prática, no Brasil, a maioria dos pacientes é automaticamente incluída no programa de hemodiálise, sem ter tido a oportunidade de escolher outra modalidade 27 .

Este estudo evidenciou a importância da atenção primária do Município de Belo Horizonte no planejamento da diálise dos pacientes diabéticos, fortalecendo este nível de atenção no cuidado do doente renal crônico, de acordo com os princípios da Política Nacional de Atenção ao Doente Renal.

Cabe ressaltar que este trabalho apresenta limitações referentes ao tipo de delineamento transversal, no qual os resultados encontrados não poderão inferir relação causal. Há a possibilidade de viés de memória, uma vez que as variáveis estudadas são autorrelatadas. Outra li- 
mitação pode estar relacionada ao viés de sobrevida seletiva, devido ao fato de que os indivíduos entrevistados são os sobreviventes da doença renal crônica terminal, que apresenta alta taxa de mortalidade.

Por outro lado, salienta-se como vantagem o fato de ter sido utilizado para a análise dados primários de todos os pacientes que iniciaram a diálise no Município de Belo Horizonte, no período de janeiro de 2006 a dezembro de 2007.

Espera-se, com base nesses resultados, subsidiar o planejamento de estratégias para o cuidado dos pacientes diabéticos atendidos pela rede SUS do Município de Belo Horizonte, com a finalidade de aprimorar o cuidado pré-diálise. A qualidade da atenção prestada ao doente renal e uma efetiva prevenção exigem que se invista em uma política de formação de profissionais, em um processo permanente de capacitação daqueles envolvidos no cuidado integral ao doente renal, além da integração dos recursos disponíveis na rede SUS. Tais são os desafios a serem enfrentados, para que se alcance a proposta da Política Nacional de Atenção Integral ao Portador de Doença Renal.

\section{Conclusão}

Os resultados encontrados evidenciam que a população estudada, diabéticos portadores de doença renal crônica terminal, tem um cuidado pré-diálise deficiente. Fica evidente que o encaminhamento precoce ao nefrologista, tãosomente, não garante o cuidado pré-diálise ideal. São necessários esforços para a articulação da rede de serviços existente e sua organização em um modelo assistencial voltado para o cidadão e para a atenção primária à saúde, além da melhoria das ações assistenciais preventivas e curativas e de uma adequada preparação da equipe de saúde que lida diariamente com pacientes portadores de risco para o desenvolvimento e agravamento da doença renal crônica. O diagnóstico precoce, o controle das complicações e da progressão da doença renal crônica poderão garantir a melhor qualidade de vida dos pacientes, além de contribuir com a redução dos gastos do SUS com a terapia renal substitutiva.

\section{Resumen}

El objetivo fue analizar los factores asociados al inicio planificado de la diálisis en los pacientes diabéticos que comenzaron el tratamiento en la ciudad de Belo Horizonte, Minas Gerais, Brasil. Se trata de un estudio transversal con 250 pacientes diabéticos que comenzaron tratamiento de diálisis entre enero de 2006 y diciembre de 2007. Iniciar la diálisis con fístula arteriovenosa o diálisis peritoneal se clasificó como inicio planificado. Se investigaron variables sociodemográficas, clínicas y de utilización de los servicios de salud por medio de una encuesta semi-estructurada. Para la regresión multivariante se utilizó Poisson. El 70\% de los pacientes iniciaron diálisis de forma no planificada y un 67\% de los que consultaron con el nefrólogo fueron remitidos al mismo tras más de 4 meses de dolencia. Asistir al centro de salud, no tener una primera consulta con un nefrólogo pagado por el SUS y contar con la opción de elección para el tratamiento de la enfermedad renal son factores relacionados con la iniciación prevista de la diálisis. La diálisis no planificada es común en la ciudad de Belo Horizonte y se produce independientemente del momento en el que se deriva al paciente al nefrólogo.

Falio Renal Crónico; Diabetes Mellitus; Diálisis; Evaluación em Salud; Estudios Transversales 


\section{Contribuição}

E. R. M. Peixoto foi responsável pela concepção do projeto, análise e interpretação dos dados e redação do artigo. I. A. Reis colaborou com a análise e interpretação dos dados e aprovação final da versão a ser publicada. E. L. Machado participou da análise de dados e aprovação final da versão a ser publicada. E. I. G. Andrade contribuiu significativamente na elaboração do rascunho e na revisão crítica do conteúdo, e participou da aprovação da versão final do manuscrito. F. A. Acurcio contribuiu significativamente na revisão crítica do conteúdo e participou da aprovação da versão final do manuscrito. M. L. Cherchiglia participou na revisão de planejamento, relevante crítica do conteúdo intelectual e aprovação final da versão a ser publicada.

\section{Agradecimentos}

M. L. Cherchiglia e F. A. Acurcio são bolsistas de produtividade do CNPq e do Programa do Pesquisador Mineiro da FAPEMIG. E. I. G. Andrade é bolsista de produtividade do CNPq.

Os autores agradecem aos integrantes do Grupo de Pesquisa em Economia da Saúde da Universidade Federal de Minas Gerais, por sua valorosa contribuição desde o desenho metodológico até a revisão final. Ao apoio financeiro do Fundo Nacional de Saúde/Ministério da Saúde (convênio no 4864/2005), CNPq (Processo 409729/2006-0) e FAPEMIG (Processo EDT 3284/06 e Processo CDS - PPM-00098-11).

\section{Referências}

1. Romão Jr. JE. Doença renal crônica: definição, epidemiologia e classificação. J Bras Nefrol 2004; 26 Suppl 1:1-3.

2. Monteiro CA, Conde WA, Popkin BM. Is obesity replacing or adding to undernutrition? Evidence from different social classes in Brazil. Public Health Nutr 2002; 5:105-12.

3. Bastos MG, Kirsztajn GM. Doença renal crônica: importância do diagnóstico precoce, encaminhamento imediato e abordagem interdisciplinar estruturada para melhora do desfecho em pacientes ainda não submetidos à diálise. J Bras Nefrol 2011; 33:93-108.

4. Cabral ALLV. Itinerários terapêuticos: compreendendo a trajetória de pessoas em hemodiálise no Sistema Único de Saúde de Belo Horizonte [Dissertação de Mestrado]. Belo Horizonte: Universidade Federal de Minas; 2010.

5. Coordenação Geral de Alta Complexidade, Secretaria de Assistência à Saúde Brasil, Ministério da Saúde. Política nacional de atenção ao portador de doença renal. Brasília: Ministério da Saúde; 2004. (Cadernos de Atenção Especializada, 2).

6. Sociedade Brasileira de Nefrologia. Censo 2011. http://www.sbn.org.br/leigos/index.php?censo (acessado em 10/Out/2012).
7. Wavamunno MD, Harris DC. The need for early nephrology referral. Kidney Int Suppl 2005; (94):S128-32.

8. Romão Jr. JE. A doença renal crônica: do diagnóstico ao tratamento. Prát Hosp (São Paulo, 1999) 2007; (52):183-7.

9. Marrón B, Ortiz A, Sequera P, Martín-Reyes G, Arriba G, Lamas JM, et al. Impact of end-stage renal disease care in planned dialysis start and type of renal replacement therapy - a Spanish multicentre experience. Nephrol Dial Transplant 2006; 21 Suppl 2:ii51-5.

10. Lorenzo V, Martin M, Rufino M, Hernández D, Torres A, Ayus JC. Predialysis nephrologic care and a functioning arteriovenous fistula at entry are associated with better survival in incident hemodialysis patients: an observational cohort study. Am J Kidney Dis 2004; 43:999-1007.

11. Mendelssohn DC, Curtis B, Yeates K, Langlois S, McRae JM, Semeniuk JM, et al. Suboptimal initiation of dialysis with and without early referral to a nephrologist. Nephrol Dial Transplant Jan 2011; 26:2959-65. 
12. Arora P, Obrador GT, Ruthazer R, Kausz AT, Meyer $\mathrm{KB}$, Jenuleson CS, et al. Prevalence, predictors and consequences of late nephrology referral at a tertiary centre. J Am Soc Nephrol 1999; 10:1281-6.

13. Cass A, Cunningham J, Snelling P, Ayanian JZ. Late referral to a nephrologist reduces access to renal transplantation. Am J Kidney Dis 2003; 42:1043-9.

14. Roderick P, Jones C, Drey N, Blakeley S, Webster P, Goddard J, et al. Late referral for end-stage renal disease: a region-wide survey in the south west of England. Nephrol Dial Transplant 2002; 17:1252-9.

15. Khan IH, Catto GR, Edward N, MacLeod AM. Chronic renal failure: Factors influencing nephrology referral. QJM 1994; 87:559-64.

16. Sesso R, Belasco AG. Late diagnosis of chronic renal failure and mortality in maintenance dialysis. Nephrol Dial Transplant 1996; 11:2417-20.

17. Heatley SA. Optimal referral is early referral. Perit Dial Int 2009; 29 Suppl 2:S128-31.

18. Araújo RB, Santos I, Cavaleti MA, Costa JSD, Béria JU. Avaliação do cuidado prestado a pacientes diabéticos em nível primário. Rev Saúde Pública 1999; 33:24-32.

19. Batista LKC, Pinheiro HS, Fuchs LC, Oliveira T, Belchior FJE, Galil AGS, et al. Manuseio da doença renal crônica em pacientes com hipertensão e diabetes. J Bras Nefrol 2005; 27:8-13

20. Ciconelli RM, Ferraz MB, Santos W, Meinão I, Quaresma MR. Tradução para a língua portuguesa e validação do questionário genérico de avaliação de qualidade de vida SF-36 (Brasil SF 36). Rev Bras Reumatol 1999; 39:143-50.

21. Duarte PS, Miyazaki MCOS, Ciconelli RM, Sesso R. Tradução e adaptação cultural do instrumento de avaliação de qualidade de vida para pacientes renais crônicos (KDQOL-SFTM). Rev Assoc Med Bras 2003; 49:375-81.

22. Gorriz JL, Sancho A, Pallardó LM, Amoedo ML, Martín M, Sanz P, et al. Prognostic significance of programmed dialysis in patients who initiate renal substitutive treatment. Multicenter study in Spain. Nefrología 2002; 22:49-59.

23. Associação Brasileira de Empresas de Pesquisa. Dados com base no levantamento sócio-econômi co - 2000. http://www.abep.org-abep@abep.org (acessado em 08/Jan/2013).

24. Barros AJD, Hirakata VN. Alternatives for logistic regression in cross-sectional studies: an empirical comparison of models that directly estimate the prevalence ratio. BMC Med Res Methodol 2003; 3:21.

25. Mendelssohn DC, Ethier J, Elder SJ, Saran R, Port FK, Pisoni RL. Haemodialysis vascular access problems in Canada: results from the Dialysis Outcomes and Practice Patterns Study (DOPPS II). Nephrol Dial Transplant 2006; 21:721-8.

26. Cherchiglia ML, Machado EL, Szuster DAC, Andrade ELG, Acúrcio FA, Caiaffa WT, et al. Perfil epidemiológico dos pacientes em terapia renal substitutiva no Brasil, 2000-2004. Rev Saúde Pública 2010; 44:639-49.

27. Andrade MV, Junoy JP, Andrade EIG, Acurcio FA, Sesso R, Queiroz OVD, et al. Allocation of initial modality for renal replacement therapy in Brazil. Clin J Am Soc Nephrol 2010; 5:637-44.
28. Nesrallah G, Mendelssohn DC. Modality options for renal replacement therapy: the integrate care concept revisited. Hemodial Int 2006; 10:143-51.

29. Wauters JP, Uehlinger D. Non-medical factors influencing peritoneal dialysis utilization: the Swiss experience. Nephrol Dial Transplant 2004; 19:1363-7.

30. Silva GM, Gomes IC, Andrade EIG, Lima EM, Acurcio FA, Cherchiglia ML. Permanent vascular access in patients with end-stage renal disease, Brazil. Rev Saúde Pública 2011; 45:241-8.

31. Pisoni RL, Young EW, Dykstra DM, Greenwood RN, Hecking E, Gillespie B, et al. Vascular access use in Europe and the United States: results from the DOPPS. Kidney Int 2002; 61:305-16.

32. Bello AK, Nwankwo E, El Nahas AM. Prevention of chronic kidney disease: a global challenge. Kidney Int Suppl 2005; 98:S11-7.

33. Prefeitura Municipal de Belo Horizonte. Política municipal de saúde. http://portalpbh.pbh.gov.br/ $\mathrm{pbh} / \mathrm{ecp} / \mathrm{contents}$.do?evento=conteudo\&idConte $\mathrm{udo}=18830 \& \mathrm{chPlc}=18830 \& \& \mathrm{pIdPlc}=\& \mathrm{app}=$ salanot icias (acessado em 20/Out/2012).

34. Prefeitura de Belo Horizonte. Estrutura da secretaria municipal de saúde. http://portalpbh.pbh.gov. $\mathrm{br} / \mathrm{pbh} / \mathrm{ecp} / \mathrm{comunidade} . \mathrm{do}$ ?evento=portlet\&pId $\mathrm{Plc}=\mathrm{ecp}$ TaxonomiaMenuPortal\&app $=$ saude $\& \operatorname{tax}=$ $7481 \&$ lang $=$ pt BR\&pg $=5571 \&$ taxp $=0 \&$ (acessado em 27/Nov/2012).

35. Machado EL, Cherchiglia ML, Acurcio FA. Perfil e desfecho clínico de pacientes em lista de espera por transplante renal, Belo Horizonte (MG, Brasil), 2000-2005. Ciênc Saúde Coletiva 2011; 16:1981-92.

36. Astor BC, Eustace JA, Powe NR, Klaq MJ, Sadler JH, Fink NE, et al. Timing of nephrologist referral and arteriovenous access use: the CHOICE Study. Am J Kidney Dis 2001; 38:494-501.

37. Roubicek C, Brunet P, Huiart L, Thirion X, Leonetti F, Dussol B, et al. Timing of nephrology referral: influence on mortality and morbidity. Am J Kidney Dis 2000; 36:35-41.

38. Lameire N, Van Biesen W, Wiedemann M. Economic issues, referral patterns, and choice of treatment modality of end-stage renal disease in Europe. In: Lameire N, Mehta R, editors. Complications of dialysis. New York: Marcel Dekker; 2000. p 753-75.

39. Pereira RPA. O itinerário terapêutico dos pacientes em TRS em Belo Horizonte: uma análise da integralidade à luz de suas percepções [Dissertação de Mestrado]. Belo Horizonte: Universidade Federal de Minas Gerais; 2008

40. Ministério da Saúde. Índice de desempenho do Sistema Único de Saúde (IDSUS). http://saladein formacao.saude.gov.br/cartogramas/sage/abre mapa.php?id=1 (acessado em 22/Out/2012).

41. Finkelstein FO, Story K, Firanek C, Barre P, Takano T, Soroka S, et al. Perceived knowledge among patients cared for by nephrologists about chronic kidney disease and end-stage renal disease therapies. Kidney Int 2008; 74:1178-84.

Recebido em 08/Nov/2012

Versão final reapresentada em 02/Fev/2013

Aprovado em 08/Fev/2013 